Published in final edited form as:

Wiley Interdiscip Rev RNA. 2012 September ; 3(5): 697-705. doi:10.1002/wrna.1129.

\title{
So You Want to Know if Your Message Has an IRES?
}

\author{
Sunnie Thompson \\ University of Alabama at Birmingham, Microbiology
}

\begin{abstract}
Transcriptional regulation of gene expression has been widely studied. More recently, there has been increasing appreciation of the role that translational regulation plays in gene expression, resulting in a number of new fields engaging in translational studies. Regulation of protein synthesis is critical for cell growth, development and survival, and is primarily controlled at the initiation step. Eukaryotic cells utilize multiple mechanisms to initiate translation, depending on cell stress, growth conditions, viral infection, or the sequences present in the mRNA. While the vast majority of mRNAs are translated in a cap-dependent manner, an important subset of mRNAs use an alternative mechanism, whereby ribosomes are recruited internally to the message to initiate cap-independent translation. Some of these mRNAs contain an internal ribosome entry site (IRES) located in the $5^{\prime}$ untranslated region (UTR). However, establishing that an RNA element is a functional IRES requires a number of carefully executed experiments with specific controls. This review will clearly explain the required experiments, and the pros and cons of various assays, used to determine whether (or not) an RNA element functions as an IRES to promote initiation of translation. We hope that demystifying the accepted methods for assaying IRES activity will open the study of this important mechanism to the broader community.
\end{abstract}

\section{INTRODUCTION}

Transcriptional regulation plays an important role in gene expression. However, not all mRNAs are created equal and translated with equal efficiency; rather, the eukaryotic cell uses multiple means to regulate gene expression at the level of protein synthesis. Translation is predominately regulated during initiation, either globally or through sequences in the mRNA that allow for message specific regulation. Translational control plays an important role in differentiation, cell growth, mitosis, angiogenesis, and apoptosis. Therefore, dysregulation of translation initiation makes a significant contribution to tumor cell growth, survival, invasion, and metastasis. ${ }^{1-5}$ Many cellular messages that encode proteins required for tumorigenesis utilize an IRES-mediated mechanism of initiating proteins synthesis. The proteins encoded by these messages are generally expressed in low amounts but can have a significant affect on the cell program, such as transcription factors. It is clear that capdependent translation is the work-horse of the cell and that it is used for proteins required in bulk, such as $\beta$-actin. However, when the cell encounters rough times (e.g. starvation, hypoxia, or apoptosis) it halts the energy demanding process of protein synthesis through a dramatic global reduction in cap-dependent translation. In order to cope with the stress or to enter into programmed cell death, the cell requires synthesis of certain proteins. These proteins may be encoded by mRNAs that contain IRESs.

This review focuses on identifying functional IRESs in mRNAs. While IRESs were first discovered in mammalian viruses ${ }^{6,7}$ it was not long before the first cellular IRES was discovered. ${ }^{8}$ Now, 10 to $15 \%$ of cellular mRNAs are estimated to contain an IRES. ${ }^{3}$ This

sunnie@uab.edu. 
estimate is based on multiple experiments under various conditions when IRES-mediated translation would be favored. ${ }^{9-12}$ These types of experiments cast a broad net, and while they provide some indication that a cellular message may contain IRES, they fall far short of the bar in demonstrating that the protein expression is IRES-dependent. This must be experimentally demonstrated for each one and a series of controls must be performed. Most of the effort that goes into establishing that an RNA element has IRES activity is spent on the control experiments that rule out the possibility that it could be anything else but IRES activity.

\section{MECHANISMS OF INITIATING TRANSLATION}

There are two basic mechanisms of initiating translation: cap-dependent and capindependent. However, there are variations on these basic mechanisms, and it is important to understand all of the mechanisms of initiating protein synthesis, as these must be ruled out in order to establish IRES activity.

\section{Cap-dependent}

The majority of eukaryotic mRNAs are translated using a cap-dependent mechanism of translation whereby 10 to 13 eukaryotic initiation factors (eIFs) function to recognize the $5^{\prime}$ cap structure on the mRNA, recruit the 40S ribosomal subunit to the $5^{\prime}$ end of the mRNA (Fig. 1a), scan down the message to locate the AUG start codon, and facilitate 60S subunit joining to form a translationally active $80 \mathrm{~S}$ ribosome. For a more detailed discussion of capdependent translation see other recent reviews. ${ }^{13,14}$ However, a brief review of the roles of these factors is necessary as several are important in IRES-mediated translation as well. In cap-dependent translation the $5^{\prime}$ cap structure is recognized by a complex that includes the cap-binding protein (eIF4E), a scaffold protein (eIF4G) and an RNA helicase (eIF4A). This complex recruits the $43 \mathrm{~S}$ pre-initiation complex (40S subunit, eIF1, eIF5, eIF1A, eIF2•mettRNA $_{i} \cdot G T P$, and eIF3) to the $5^{\prime}$ proximal region of the mRNA. The $40 \mathrm{~S}$ subunit scans in a $5^{\prime}$ to $3^{\prime}$ direction along the message until the start codon, AUG, is recognized and GTP is hydrolyzed. The $60 \mathrm{~S}$ subunit then joins to form an $80 \mathrm{~S}$ complex. ${ }^{15}$ This complex process provides both efficiency for initiation and multiple avenues for the cell to regulate translation globally or in a message specific manner. ${ }^{14}$

Leaky Scanning, uORFs, and ribosomal shunting-In vertebrates the first AUG in a good Kozak consensus sequence context ( $\left.5^{\prime}-\mathrm{GCCGCC}(\mathrm{A} / \mathrm{G}) \mathrm{CCAUGG}-3^{\prime}\right)$ that is encountered by the scanning ribosome is used for initiating protein synthesis. ${ }^{16} \mathrm{mRNA}$ specific regulation can occur by leaky scanning (Fig 1b) through an upstream start codon that is in a sub-optimal context ${ }^{17}$, or by the presence of upstream open reading frames (uORFs) that are upstream of the main protein coding sequence (Fig. 1d). ${ }^{15}$ The short uORFs allow for continued scanning of the $40 \mathrm{~S}$ ribosomal subunit after termination of the stop codon. When ternary complexes (eIF2•met-tRNA $\left.\bullet_{\mathrm{i}} \mathrm{GTP}\right)$ are low then the scanning $40 \mathrm{~S}$ subunit takes longer to acquire a new ternary complex (required in order to recognize a start codon), and it can bypass other start codons (uORFs) until a ternary complex is obtained. A percentage of 40S subunits will acquire the ternary complex before reaching the start codon for the main coding sequence and initiate protein synthesis. ${ }^{15,18}$

Ribosome shunting or reinitiation involves discontinuous scanning from a cap-dependent recruitment of the ribosome (Fig. 1C). The ribosome is recruited to the $5^{\prime}$ end of the message in a cap-dependent manner, the $40 \mathrm{~S}$ subunit may or may not translate a short uORF, then a $5^{\prime}$ donor site transfers or shunts the $40 \mathrm{~S}$ subunit to a $3^{\prime}$ acceptor site, bypassing a large segment of mRNA (without scanning through it) to initiate translation at a downstream AUG. ${ }^{19}$ The molecular mechanisms of ribosomal shunting are poorly understood and no defined sequence or RNA structure has been universally associated with shunting. 


\section{Cap-independent}

Cap-independent translation is just that, initiation of protein synthesis that does not require a $5^{\prime}$ cap structure. Two mechanisms have been described that are mediated by either IRESs or cap-independent translation elements (CITEs). ${ }^{20,}{ }^{21}$ IRESs function by recruiting a ribosome internally to an mRNA (Fig. 2a). The IRES can bind to initiation factors, which in turn recruit ribosome subunits (e.g. picornaviral IRESs), it can bind directly to the ribosomal subunit (e.g. cricket paralysis virus intergenic region and hepatitis $\mathrm{C}$ virus IRESs), or it can base pair with rRNA sequences ${ }^{22-24}$ similar to shine-Dalgarno sequences for prokaryotic initiation. It is unclear whether CITEs are a separate mechanism or a variation of an IRES. Typically CITEs are located in the $3^{\prime}$ UTR, but they can function efficiently in the $5^{\prime}$ UTR as well, suggesting that they provide a mechanism to increase the concentration of ribosomes proximal to the start codon, whether they are in the $5^{\prime}$ UTR or are brought into proximity of the $5^{\prime}$ UTR by circularization of the mRNA through long distance base pairing. CITEs function to recruit the translational machinery to the RNA by binding eIF4G (the scaffold protein) or/and eIF4E (the cap-binding protein) (Fig. 2b). So far CITEs have only been identified in plant RNA viruses. ${ }^{21}$ It remains unclear whether these CITEs can function as IRESs or if they require a free $5^{\prime}$ end. The translation field awaits generation of a circular RNA (see below) with a CITE element.

\section{WHAT IS AN IRES?}

An IRES is defined by a functional assay that establishes that an RNA element or sequence is capable of recruiting a ribosome internally to the message. IRESs can only be identified experimentally. There is no consensus sequence or RNA structure that defines an IRES. ${ }^{25}$ IRESs cannot be predicted bioinformatically. ${ }^{26}$ In fact, both short unstructured sequences and long structured 5'UTRs have been demonstrated to have IRES activity. ${ }^{23}, 27$ That being said, the following properties may suggest that an mRNA contains an IRES: 1) If it contains multiple unused upstream start codons (this could also indicate regulation by uORFs (Fig. 1d), as illustrated by the yeast GCN4 $\mathrm{mRNA}^{28}$ ). 2) If it is long and/or structured; this decreases the efficiency of scanning and may suggest there is an IRES within the $5^{\prime}$ UTR. 3) If the protein is expressed under stress or conditions when cap-dependent translation is decreased without corresponding changes in transcript abundance. However, none of these factors can establish whether on not an mRNA contains an IRES, rather a functional assay must be performed along with several control experiments (detailed below).

Much more is understood about viral IRESs and thus parallels are often drawn between viral and cellular IRESs. They may have some similarities with respect to mechanism, but their purposes are quite different. Viral IRESs are highly structured and the goal is to generate as much viral proteins as possible, therefore these IRESs are generally (but not always) some of the most active IRESs known. A cellular IRES, however, can allow continued or induced expression of a protein when cap-dependent translation is suppressed. Therefore, a cellular mRNA that contains an IRES may use cap-dependent translation and only switch to IRESmediated translation when cap-dependent translation is diminished. Alternatively, a cellular mRNA may be present in the cell but translated only when cap-dependent translation is down-regulated and IRES-mediated translation prevails. The fact that cellular messages can use both mechanisms of initiating translation makes it difficult to separate out the contributions of the different mechanisms of translation that are being used in a population. Therefore, this necessitates introducing the $5^{\prime}$ UTR into an IRES reporter (see below) to evaluate whether it is capable of IRES-mediated translation. 


\section{EVALUATING IRES ACTIVITY}

\section{Bicistronic reporter assay}

Generally the negative result is the hardest to prove in science, but this is not the case for evaluating IRES activity. It is much easier to do a single experiment and rule out the possibility of an IRES than to conclusively demonstrate IRES activity. The most common and easiest method used test for IRES activity is to clone the putative IRES sequences into a DNA bicistronic reporter and assay for expression of the second cistron (Fig 3a). The cistrons can be any protein that can be easily quantified; commonly used reporters are Renilla and firefly luciferase, and $\beta$-gal. We have observed that optimal translation of the bicistronic reporter occurs at 24 and up to 48 hours after transient transfection of the DNA plasmid into tissue culture cells. The expression of the first cistron will be cap-dependent while expression of the second cistron may be IRES-dependent. Cap-dependent translation is very efficient and the relative activity of the cap-dependent reporter will be much higher (typically several logs higher) than expression of the second cistron. A control (empty) bicistronic reporter should be used for comparison and establishing baselines. Bicistronic reporters may have some "stuffer" structured sequences that prevent readthrough of the stop codon of the first cistron to translate the second cistron. These sequences can either be structured RNA sequences that do not have IRES activity such as the $\triangle \mathrm{EMCV}$ IRES sequence $^{29}$ or have additional stop codons and sequences that place the first cistron out of frame of the second cistron. The goal here is to reduce background expression of the second cistron (due to readthrough of the stop codon or continued scanning of the ribosome) as much as possible. If the control bicistronic reporter exhibits high background levels of translation (compared to assays without reporter), then, steps can be taken to reduce the background level of expression of the second cistron (discussed above).

If the bicistronic reporter assay demonstrates that expression of the second cistron is similar to the control (empty) bicistronic reporter, then there is likely no IRES activity and translational regulation is most likely through a different mechanism. If no IRES activity is detected in the bicistronic reporter assay then this is generally the end of experiment as long as the initiating AUG start codon is in frame with the reporter coding region. Some IRESs initiate translation by scanning down to the AUG however, some assemble the 40S subunit directly at the start codon. Therefore, position of the AUG relative to the IRES could be important (and potentially the first few codons after the AUG). However, to rigorously rule out the possibility of an IRES, sequences downstream of the IRES that include a portion of the coding region should be included since some times these can contribute to IRES activity. Alternatively, if the putative IRES sequences result in a significantly higher level of expression of the second cistron over the control, then this may indicate that an IRES is present. However, additional experiments and controls will be required to establish that expression of the second cistron is not due to a technical artifact such as cryptic promoter activity or introduction of a cryptic splice site (see below).

Circular RNA-The bicistronic reporter assay is the most commonly used assay to test for IRES activity, however there are other assays that are perfectly acceptable, such as insertion of the putative IRES sequences into a circular RNA. Since cap-dependent translation requires a free $5^{\prime}$ end, generation of a circular RNA that lacks a free $5^{\prime}$ end can be used to demonstrate that ribosomes are recruited internally to the mRNAs (Fig 3b). Therefore, if translation occurs, it must be by internal initiation. Briefly, an RNA containing the putative IRES and a downstream reporter protein is generated by in vitro transcription and circularized using a bridging oligo that brings the $5^{\prime}$ and $3^{\prime}$ ends together. T4 DNA ligase is used to ligate the ends together. Circularized RNAs can be gel isolated based on their reduced gel mobility relative to their linear counterparts. ${ }^{30,31}$ The most rigorous 
demonstration using a circular RNA is to eliminate all stop codons such that the ribosomes will translate around the circular RNA multiple times to generate a long repetitive polyprotein chain (Fig. 3b). This demonstrates that translation did not occur on broken RNAs. A single protease cleavage site can be introduced to the sequence and upon addition of the protease, the polyprotein should collapse down in to a single protein equivalent in size to the ribosome translating once around the circle. ${ }^{30}$ While protein expression from a circular RNA is definitive evidence of an IRES, this approach is technically more difficult and may suffer from some of the same caveats listed below for RNA transfections that may result in a false negative.

5'hairpin - A third assay that may be used to evaluate IRES activity is to introduce a stable hairpin structure at the $5^{\prime}$ end of the mRNA to block cap-dependent translation. ${ }^{8}$ The hairpin blocks cap-dependent translation but should not have an effect on IRES-mediated translation. In principle a $5^{\prime}$ hairpin could be introduced to either a bicistronic or monocistronic reporter. In a monocistronic construct, cap-dependent translation of a containing reporter would not be inhibited (comparison of translational activity of a reporter containing the putative IRES element with and without the hairpin). Addition of a $5^{\prime}$ hairpin to a cellular mRNA is an effective assay to detect IRES activity such that minimal changes are made to the sequence context of the IRES. A potential caveat of this approach is that if the hairpin is not introduced close enough to the $5^{\prime}$ end or is not stable enough, then it will not fully inhibit cap-dependent translation. Therefore, it is essential that a control reporter is generated that has a cap-dependent $5^{\prime}$ UTR (such as $\beta$-actin) to demonstrate that the hairpin completely blocks cap-dependent translation. In a bicistronic context, translation of the first cistron would be inhibited by the hairpin, but translation of the second cistron by an IRES would be unaffected. The bicistronic hairpin reporter would be compared to expression from an identical reporter lacking the hairpin.

RNA IRES Reporters-It is not uncommon for an RNA bicistronic reporter to be used to assess IRES activity. The RNA reporter can be synthesized in vitro and transfected into mammalian cells. While RNA transfections work well for viral IRESs, there have been reports that suggest that cellular IRESs require a "nuclear experience" in order to be functional. ${ }^{32,33}$ The controversy over this is still not settled in the translation field. There is still no clear evidence for how this nuclear experience "activates" the cellular IRES. The possibilities may include modification of the mRNA (by methylation, pseudouridylation or other mRNA modifications) or association of an RNA-binding protein. RNA binding proteins termed IRES transacting factors (ITAFs) are required by certain IRESs for activity (for review see ${ }^{34}$ ). In general, ITAFs are either exclusively nuclear or proteins that are known to cycle between the nucleus and cytoplasm. ${ }^{35}$ Very few are located exclusively in the cytoplasm where IRESs are active. This argues that the IRES might need to pass through the nucleus to acquire these proteins. The other side in this controversy takes the fact that certain IRESs are more active in DNA than in RNA reporters as proof that "IRES" activity is really caused by cryptic promoters or aberrant splicing events in the nucleus. While this criticism is clearly warranted for certain studies that have mistakenly assigned IRES activity when in fact there is not an IRES, it is clear that certain cellular mRNAs and viral RNAs do contain a functional IRES. ${ }^{36}$ Justified criticisms of studies with insufficient controls cannot be used to generalize to all cellular IRES studies.

\section{In Vitro Assays for IRES activity}

Up to this point we have only discussed in vivo assays, but there are several commercially available or published methods to generate translationally active extracts. The most popular and easy to use is the rabbit reticulocyte lysate (RRL). The caveat of using this system is that RRL was designed to maximally translate any mRNA that is introduced to it. Therefore, 
non-specific initiation of translation can occur that does not rely on a $5^{\prime}{ }^{7 m} \mathrm{GpppG}$ cap or a poly(A) tail. Therefore, controls need to be in place to demonstrate specific initiation. Furthermore, some cellular and viral IRESs do not translate efficiently in cell-free translation systems. Finally, it is worth mentioning that some IRES function efficiently in RRL only if it is supplemented with a ribosomal salt-wash fraction from HeLa cells to supply the ITAFs. ${ }^{37}$

That being said, there are several good vitro translation assays that add supporting evidence for (or against) an IRES. In particular, the eIF4E (cap-binding protein) can be depleted or competed for by adding excess cap-structure to translationally active HeLa cell lysates; since IRESs do not require eIF4E (with the exception of hepatitis A virus) ${ }^{38}$, depletion should not affect IRES-mediated translation. In addition, many IRESs function when eIF4G (scaffold) is cleaved, as poliovirus does to shut-off cap-dependent translation. Thus, an increased or equivalent translation when eIF4G is cleaved by addition of a $2 \mathrm{~A}$ protease is good supporting evidence for IRES activity.

\section{Eliminating technical reasons for second cistron expression from a bicistronic reporter}

If expression of the second cistron in the bicistronic reporter has been observed, the next step is to rule out other, non-IRES dependent causes, such as cryptic promoter activity, activation of a splicing event, readthrough, or ribosomal shunting. The most common false positive is caused by cryptic promoter activity.

Ruling out cryptic promoters-A cryptic promoter can generate a separate, monocistronic mRNA, that contains the second cistron expressed through the cap-dependent mechanism (Fig. 4a). Importantly, this transcript can be present in addition to the full-length bicistronic reporter. Therefore the control must not merely confirm that the full-length bicistronic reporter is expressed, but demonstrate that no smaller transcripts are expressed that could produce a functional second cistron protein. There are two methods to rule out cryptic promoters. The first and most standard method is a northern analysis to detect all RNA transcripts generated. The probe should target sequences in the second cistron that are essential for reporter activity in order to detect shorter transcripts that may be responsible for or contribute to the expression of the second cistron. Even a small amount of monocistronic RNA from a weak cryptic promoter would result in a significant level of expression of the second cistron by a cap-dependent mechanism. Therefore, the signal from the northern must be sufficiently intense (or even over exposed) to detect a low level of signal from a cryptic promoter. That being said, it has been our experience that cryptic promoter activity is easily detected by northern analysis.

An alternative to northern analysis has been to perform an RT-PCR (reverse transcription polymerase chain reaction) to detect shorter transcripts. Another PCR approach that has been used uses real-time PCR to compare the RNA levels of the first cistron and second cistrons. If their levels are equivalent it is taken as evidence that only a full-length bicistronic mRNA is produced. However, we caution the reader that PCR only detects what is predicted to be there based on the primers used. There is a significant risk that PCR may miss unexpected transcripts. RNase protection assays are 10-fold more sensitive than northerns. However, RNase protection assays suffer from the same issues as RT-PCR. Namely, that unless the aberrant transcript is correctly anticipated it may be missed. In other words, the RNase protection assay can be very useful in determining which transcripts are present that hybridize to the probe but it does not detect transcripts that may be there but are not recognized by the probe. Identifying any unanticipated transcripts is essential to rule out false positives for IRES activity. An RNase protection assay may be used to identify sequences in an aberrant transcript to determine if it is the result of cryptic promoter or 
splicing. Therefore, we believe that northern analysis is the most rigorous test for cryptic promoters and cryptic splice sites. Given that the most common explanation for second cistron expression that is a cryptic promoter in the bicistronic reporter, the first control we perform is a northern.

Richard Lloyd has developed an RNAi assay to evaluate the transcripts from a bicistronic DNA reporter. ${ }^{39}$ Briefly, an siRNA that targets the first cistron or a control siRNA is transfected into the reporter cells with the bicistronic reporter. If only intact bicistronic mRNA is generated, then both cistrons will be affected equally. Thus, cryptic promoter activity is indicated when the second cistron is not knocked-down to the same degree as the first cistron. However, this approach cannot determine the cause of these aberrant transcripts, nor can it detect contributions from a spliced transcript that links both cistrons or readthrough of the stop codon from the first cistron. Therefore, as stated by the authors themselves, this assay must be performed in combination with other experiments such as a northern analysis and a promoter-less reporter. ${ }^{39}$

Experimental proof of the absence of a cryptic promoter is obtained by transfection of a promoter-less DNA reporter into mammalian cells. In the absence of a promoter no mRNA is generated thus no protein expression should be detected. If no second cistron activity is detected, that argues against a cryptic promoter, and thus for an IRES. However, if activity from the second cistron reporter is detected, this suggests that a cryptic promoter is present, and thus second cistron expression may be through a cap-dependent mechanism.

Ruling out splicing, readthrough, and shunting-An IRES will promote expression of the second cistron independent of whether the first cistron is translated or not. However, if expression of the second cistron results from an aberrant splicing event, ribosomal shunting, or readthrough of the upstream stop codon, then inhibiting translation of the first cistron would have a dramatic effect on second cistron expression. There are several methods to inhibit cap-dependent translation of the first cistron. However, the cleanest is to introduce a stable stem loop structure near the $5^{\prime}$ end of the bicistronic reporter. This provides steric hindrance to inhibit loading of the $40 \mathrm{~S}$ subunit at the $5^{\prime}$ end of the bicistronic message. If the hairpin inhibits translation only of the first cistron, that argues that an IRES is independently driving the second cistron. However, if the second cistron is also inhibited, then splicing, readthrough or ribosomal shunting may be contributing some, if not all, of the second cistron expression from the cap-dependent mechanism.

Another assay to demonstrate independent of expression of the second cistron expression blocks cap-dependent translation through the use of an A-cap (ApppA). While the A-cap functions to stabilize the mRNA in cells, the cap-binding protein, eIF4E, does not recognize the A-cap structure and therefore the mRNA is not a substrate for cap-dependent translation. However, this requires using an RNA reporter and therefore all the caveats of using an RNA reporter listed above would apply.

\section{Conclusion}

It has long been assumed that changes in transcript level are a good predictor of changes in protein level and thus many studies in genetics, cancer and developmental biology have focused on the transcriptome with the assumption that this accurately reflects changes in gene expression. However, a recent study comparing transcript levels with protein levels demonstrated that, in mammals, there is not a good correlation between them. ${ }^{40}$ This suggests that we have yet to appreciate the large amount of post-transcriptional regulation that contributes to gene expression. 
In the last decade, more and more researchers outside the translation field have noticed that the protein levels of their favorite protein changes, while the level of the transcript remains unchanged. This has led an increasing number of investigators to ask whether their message is translationally controlled, and then, to ask whether it may have an IRES. While the bicistronic reporter assay for assessing IRES activity is simple and elegant, the devil is in the details. Those details are the extensive number of careful controls that must be done in order to establish genuine IRES activity by positively ruling out all alternatives. Failing to perform the required controls that eliminate alternative explanations for second cistron expression, specifically cryptic promoter activity, alternate splice site use, shunting or readthrough will almost certainly result in false attribution of IRES activity. It is the hope that this review has laid out the experiments and the justifications clearly enough to allow both novice and expert to reliably establish whether or not an RNA element has real IRES activity.

\section{Acknowledgments}

I would like to thank R. Curtis Hendrickson for critical reading of the manuscript and Donald M. Dempsey for help with the figures. The research in this laboratory is supported by the National Institutes of Health (R01GM084547) and the UAB Cancer Center HIV-Associated Malignancy pilot research grant (UAB Comprehensive Cancer Center Core Support Grant P30 CA13148).

\section{References}

1. Stumpf CR, Ruggero D. The cancerous translation apparatus. Curr Opin Genet Dev. 2011; 21:474483. [PubMed: 21543223]

2. Silvera D, Formenti SC, Schneider RJ. Translational control in cancer. Nat Rev Cancer. 2010; 10:254-266. [PubMed: 20332778]

3. Spriggs KA, Stoneley M, Bushell M, Willis AE. Re-programming of translation following cell stress allows IRES-mediated translation to predominate. Biol Cell. 2008; 100:27-38. [PubMed: 18072942]

4. Le Quesne JP, Spriggs KA, Bushell M, Willis AE. Dysregulation of protein synthesis and disease. J Pathol. 2010; 220:140-151. [PubMed: 19827082]

5. Holcik M. Targeting translation for treatment of cancer--a novel role for IRES? Curr Cancer Drug Targets. 2004; 4:299-311. [PubMed: 15134536]

6. Jang SK, Krausslich HG, Nicklin MJ, Duke GM, Palmenberg AC, Wimmer E. A segment of the 5' nontranslated region of encephalomyocarditis virus RNA directs internal entry of ribosomes during in vitro translation. J Virol. 1988; 62:2636-2643. [PubMed: 2839690]

7. Pelletier J, Sonenberg N. Internal initiation of translation of eukaryotic mRNA directed by a sequence derived from poliovirus RNA. Nature. 1988; 334:320-325. [PubMed: 2839775]

8. Macejak DG, Sarnow P. Internal initiation of translation mediated by the $5^{\prime}$ leader of a cellular mRNA. Nature. 1991; 353:90-94. [PubMed: 1652694]

9. Johannes G, Carter MS, Eisen MB, Brown PO, Sarnow P. Identification of eukaryotic mRNAs that are translated at reduced cap binding complex eIF4F concentrations using a cDNA microarray. Proc Natl Acad Sci U S A. 1999; 96:13118-13123. [PubMed: 10557283]

10. Bushell M, Stoneley M, Kong YW, Hamilton TL, Spriggs KA, Dobbyn HC, Qin X, Sarnow P, Willis AE. Polypyrimidine tract binding protein regulates IRES-mediated gene expression during apoptosis. Mol Cell. 2006; 23:401-412. [PubMed: 16885029]

11. Qin X, Sarnow P. Preferential translation of internal ribosome entry site-containing mRNAs during the mitotic cycle in mammalian cells. J Biol Chem. 2004; 279:13721-13728. [PubMed: 14739278]

12. Thomas JD, Johannes GJ. Identification of mRNAs that continue to associate with polysomes during hypoxia. Rna. 2007; 13:1116-1131. [PubMed: 17488873]

13. Sonenberg N, Hinnebusch AG. Regulation of translation initiation in eukaryotes: mechanisms and biological targets. Cell. 2009; 136:731-745. [PubMed: 19239892]

14. Jackson RJ, Hellen CU, Pestova TV. The mechanism of eukaryotic translation initiation and principles of its regulation. Nat Rev Mol Cell Biol. 2010; 11:113-127. [PubMed: 20094052]

Wiley Interdiscip Rev RNA. Author manuscript; available in PMC 2013 September 01. 
15. Hinnebusch AG. Molecular mechanism of scanning and start codon selection in eukaryotes. Microbiol Mol Biol Rev. 2011; 75:434-467. first page of table of contents. [PubMed: 21885680]

16. Kozak M. An analysis of $5^{\prime}$-noncoding sequences from 699 vertebrate messenger RNAs. Nucleic Acids Res. 1987; 15:8125-8148. [PubMed: 3313277]

17. Kozak M. Point mutations define a sequence flanking the AUG initiator codon that modulates translation by eukaryotic ribosomes. Cell. 1986; 44:283-292. [PubMed: 3943125]

18. Hinnebusch AG. Gene-specific translational control of the yeast GCN4 gene by phosphorylation of eukaryotic initiation factor 2. Mol Microbiol. 1993; 10:215-223. [PubMed: 7934812]

19. Ryabova LA, Hohn T. Ribosome shunting in the cauliflower mosaic virus $35 \mathrm{~S}$ RNA leader is a special case of reinitiation of translation functioning in plant and animal systems. Genes Dev. 2000; 14:817-829. [PubMed: 10766738]

20. Hellen CU, Sarnow P. Internal ribosome entry sites in eukaryotic mRNA molecules. Genes Dev. 2001; 15:1593-1612. [PubMed: 11445534]

21. Miller WA, Wang Z, Treder K. The amazing diversity of cap-independent translation elements in the $3^{\prime}$-untranslated regions of plant viral RNAs. Biochem Soc Trans. 2007; 35:1629-1633. [PubMed: 18031280]

22. Hu MC, Tranque P, Edelman GM, Mauro VP. rRNA-complementarity in the $5^{\prime}$ untranslated region of mRNA specifying the Gtx homeodomain protein: evidence that base- pairing to $18 \mathrm{~S}$ rRNA affects translational efficiency. Proc Natl Acad Sci U S A. 1999; 96:1339-1344. [PubMed: 9990025]

23. Chappell SA, Edelman GM, Mauro VP. A 9-nt segment of a cellular mRNA can function as an internal ribosome entry site (IRES) and when present in linked multiple copies greatly enhances IRES activity. Proc Natl Acad Sci U S A. 2000; 97:1536-1541. [PubMed: 10677496]

24. Dresios J, Chappell SA, Zhou W, Mauro VP. An mRNA-rRNA base-pairing mechanism for translation initiation in eukaryotes. Nat Struct Mol Biol. 2006; 13:30-34. [PubMed: 16341227]

25. Baird SD, Lewis SM, Turcotte M, Holcik M. A search for structurally similar cellular internal ribosome entry sites. Nucleic Acids Res. 2007; 35:4664-4677. [PubMed: 17591613]

26. Baird SD, Turcotte M, Korneluk RG, Holcik M. Searching for IRES. RNA. 2006; 12:1755-1785. [PubMed: 16957278]

27. Coldwell MJ, deSchoolmeester ML, Fraser GA, Pickering BM, Packham G, Willis AE. The p36 isoform of BAG-1 is translated by internal ribosome entry following heat shock. Oncogene. 2001; 20:4095-4100. [PubMed: 11494137]

28. Hinnebusch AG. Translational regulation of GCN4 and the general amino acid control of yeast. Annu Rev Microbiol. 2005; 59:407-450. [PubMed: 16153175]

29. Carter MS, Sarnow P. Distinct mRNAs that encode La autoantigen are differentially expressed and contain internal ribosome entry sites. J Biol Chem. 2000; 275:28301-28307. [PubMed: 10871624]

30. Chen CY, Sarnow P. Initiation of protein synthesis by the eukaryotic translational apparatus on circular RNAs. Science. 1995; 268:415-417. [PubMed: 7536344]

31. Chen CY, Sarnow P. Internal ribosome entry sites tests with circular mRNAs. Methods Mol Biol. 1998; 77:355-363. [PubMed: 9770681]

32. Stoneley M, Subkhankulova T, Le Quesne JP, Coldwell MJ, Jopling CL, Belsham GJ, Willis AE. Analysis of the c-myc IRES; a potential role for cell-type specific trans-acting factors and the nuclear compartment. Nucleic Acids Res. 2000; 28:687-694. [PubMed: 10637319]

33. Semler BL, Waterman ML. IRES-mediated pathways to polysomes: nuclear versus cytoplasmic routes. Trends Microbiol. 2008; 16:1-5. [PubMed: 18083033]

34. King HA, Cobbold LC, Willis AE. The role of IRES trans-acting factors in regulating translation initiation. Biochem Soc Trans. 2010; 38:1581-1586. [PubMed: 21118130]

35. Pacheco A, Martinez-Salas E. Insights into the biology of IRES elements through riboproteomic approaches. J Biomed Biotechnol. 2010; 2010:458927. [PubMed: 20150968]

36. Schneider R, Agol VI, Andino R, Bayard F, Cavener DR, Chappell SA, Chen JJ, Darlix JL, Dasgupta A, Donze O, et al. New ways of initiating translation in eukaryotes. Mol Cell Biol. 2001; 21:8238-8246. [PubMed: 11710333] 
37. Brown BA, Ehrenfeld E. Translation of poliovirus RNA in vitro: changes in cleavage pattern and initiation sites by ribosomal salt wash. Virology. 1979; 97:396-405. [PubMed: 224589]

38. Ali IK, McKendrick L, Morley SJ, Jackson RJ. Activity of the hepatitis A virus IRES requires association between the cap-binding translation initiation factor (eIF4E) and eIF4G. J Virol. 2001; 75:7854-7863. [PubMed: 11483729]

39. Van Eden ME, Byrd MP, Sherrill KW, Lloyd RE. Demonstrating internal ribosome entry sites in eukaryotic mRNAs using stringent RNA test procedures. RNA. 2004; 10:720-730. [PubMed: 15037781]

40. Ghazalpour A, Bennett B, Petyuk VA, Orozco L, Hagopian R, Mungrue IN, Farber CR, Sinsheimer J, Kang HM, Furlotte N, et al. Comparative analysis of proteome and transcriptome variation in mouse. PLoS Genet. 2011; 7:e1001393. [PubMed: 21695224] 
(a) Cap-dependent initiation

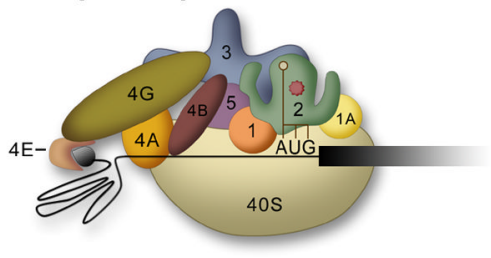

(b) Leaky scanning

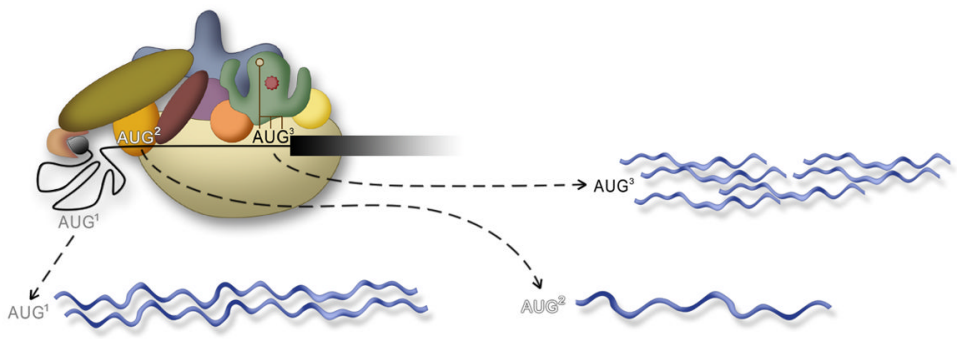

(c) Ribosome shunting or re-initiation

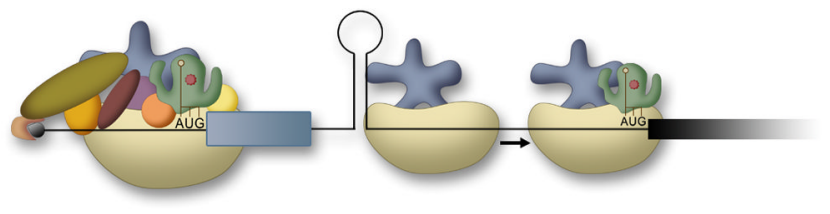

(d) UORFs

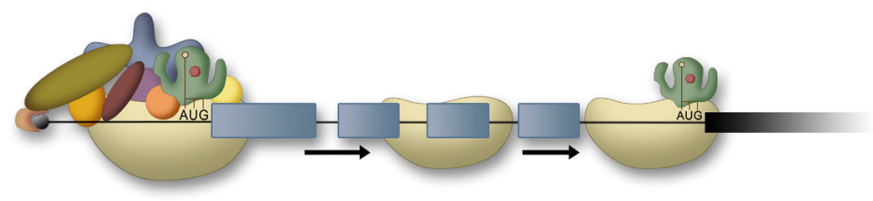

Figure 1.

Mechanisms of cap-dependent translation. (a) The standard cap-dependent mechanism involves recognition of the $5^{\prime}$ cap (black) by a complex of eukaryotic initiation factors (eIF) eIF4E, eIF4G, and eIF4A that recruit the 43S pre-initiation complex (40S subunit, eIF1, eIF5, eIF1A, eIF2•met-tRNA ${ }_{i} \cdot G T P$, and eIF3. This generates a $48 \mathrm{~S}$ complex and the $40 \mathrm{~S}$ ribosomal subunit scans down to the first AUG. The 60S subunit then joins and protein synthesis begins (not shown). (b) In leaky scanning, the $40 \mathrm{~S}$ subunit can bypass AUGs that are in a poor sequence context to generate proteins (blue) that have alternate $\mathrm{N}$-terminal ends. Here, the darker the AUG, the better the sequence context, and thus the more likely that it will be used to initiate protein synthesis. (c) Ribosome shunting requires recruitment of the ribosome through a cap-dependent mechanism as in (a), then, following scanning or translation of a short ORF the ribosome is shunted (no scanning) downstream to initiate protein synthesis. (d) The presence of uORFs can prevent translation of the major coding region unless levels of the eIF $2 \bullet$ met-tRNA ${ }_{i} \bullet \mathrm{GTP}$ are low. The ribosome is brought to the $5^{\prime}$ end as in (a), then following translation of the first uORF the 40S subunit remains 
associated with the mRNA and continues to scan down the mRNA until it acquires another eIF2•met-tRNA ${ }_{\mathrm{i}} \bullet \mathrm{GTP}$, enabling it to initiate translation at the next ORF. 


\section{(a) IRES (internal ribosome entry site)}

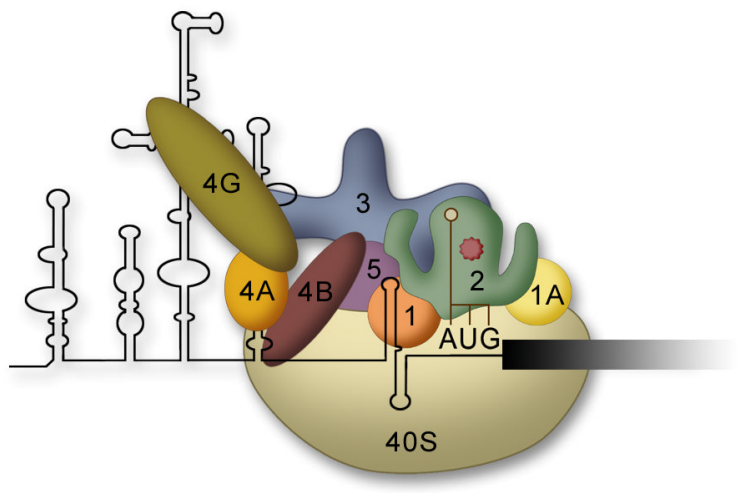

(b) CITE (Cap-independent translation element)

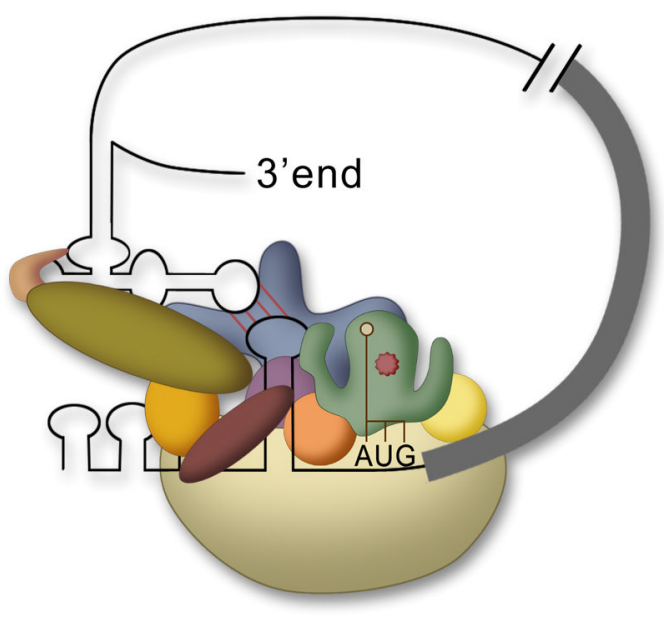

Figure 2.

Mechanisms of cap-independent translation. (a) IRESs recruit the 40S subunit internally to the mRNA using anywhere from all to none of the translation initiation factors (defined in Fig. 1a). (b) A CITE located in the $3^{\prime}$ UTR binds to the cap-binding complex (eIF4E, eIF4G, and eIF4A) and through circularization of the mRNA recruits a $43 \mathrm{~S}$ pre-initiation complex to $5^{\prime}$ UTR of the mRNA. 


\section{(a) Bicistronic reporter}

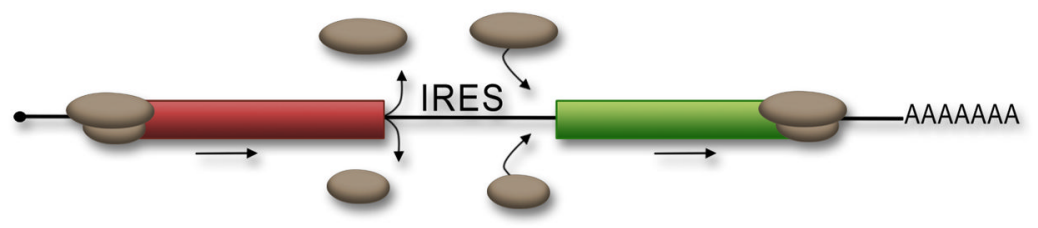

(b) Circular RNA

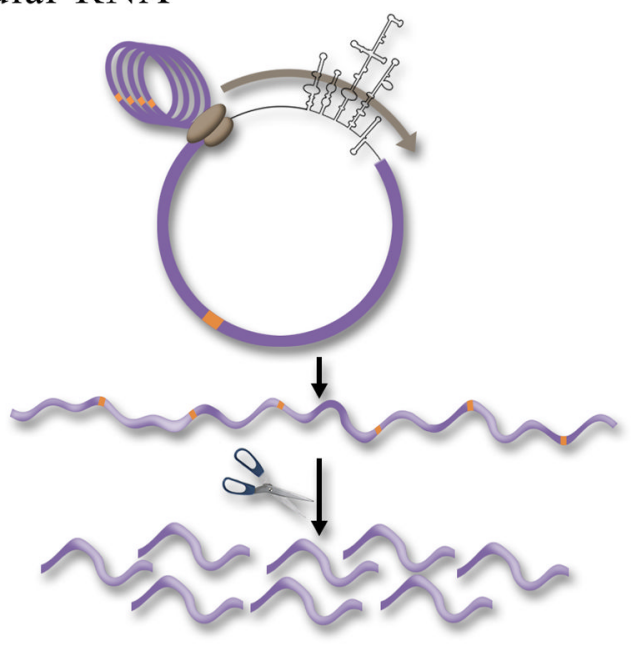

\section{(c) Hairpin reporter}

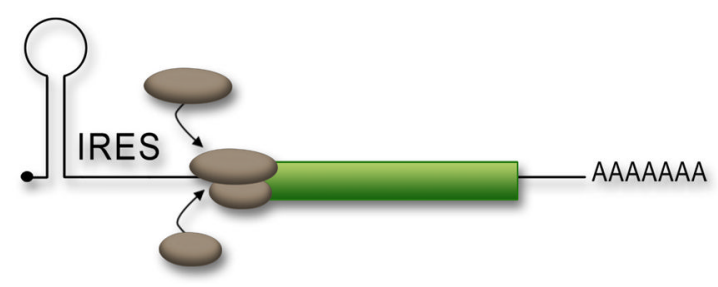

\section{Figure 3.}

Reporter assays used to assess IRES activity. (a) In the bicistronic reporter assay the IRES is placed between two cistrons. Expression of the first cistron (red) is cap-dependent whereas expression of the second cistron (green) is dependent upon a functional IRES. The ribosomal subunits are shown (brown). (b) Insertion of an IRES into a circular RNA ensures that expression of the reporter is due to internal initiation since there is no free $5^{\prime}$ end. The removal of all the stop codons in the circular RNA allows for the generation of a single continuous ORF, such that multiple rounds of translation around the circle demonstrates that the circle is intact. Insertion of a protease cleavage site (orange) allows for the collapse of the polyprotein into a single protein product consistent with the ribosome transversing around the circle once. (c) Insertion of a stable hairpin in the $5^{\prime}$ UTR can be used to block cap-dependent translation. Therefore translation of the cistron would be dependent upon a functional IRES upstream of the reporter ORF. 


\section{(a) Cryptic promoter activity}

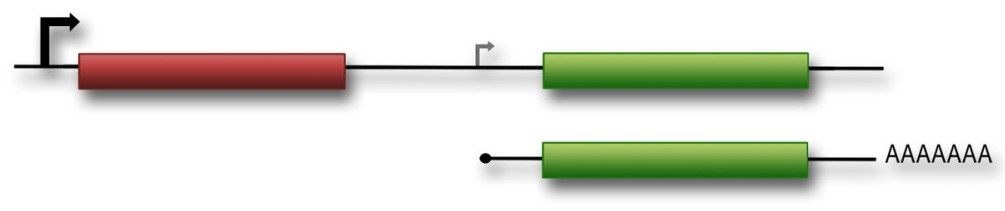

(b) Readthrough

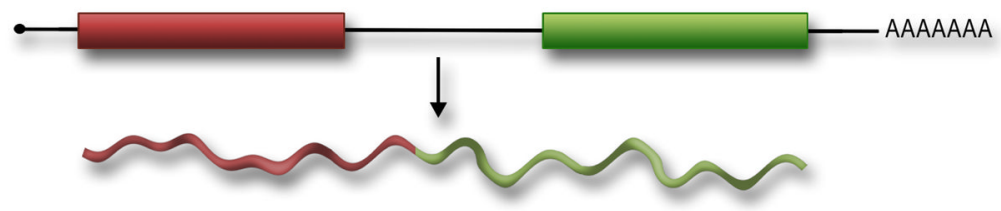

(c) Activation of a cryptic splice site

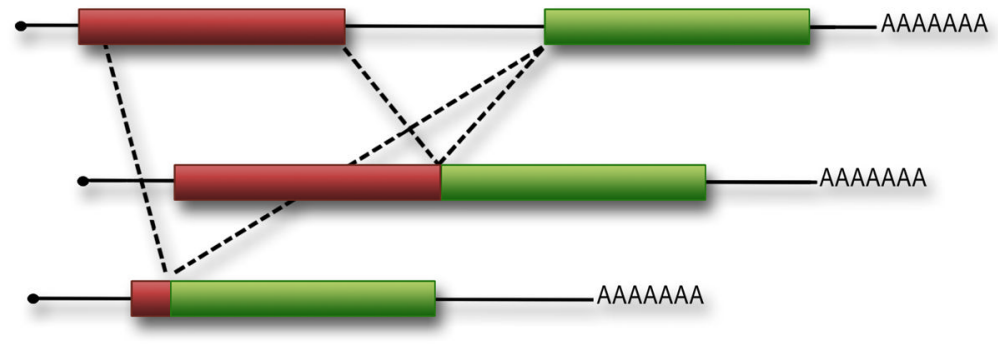

Figure 4.

Controls for the bicistronic reporter assay must be performed to rule out all possible reasons for second cistron expression aside from IRES activity. (a) The sequences inserted within the bicistronic reporter may contain weak or cryptic promoter activity that would result in the expression of a monocistronic mRNA that can be translated by the cap-dependent mechanism. (b) Readthrough of the stop codon in the first cistron would generate a chimeric reporter protein that would be translated using a cap-dependent mechanism. (c) The inserted sequences in the bicistronic reporter may contain a cryptic splice site that would generate either a chimeric protein (as in (b)) or a monocistronic RNA that encodes the second cistron. These spliced products would result in cap-dependent expression of the second cistron.

Wiley Interdiscip Rev RNA. Author manuscript; available in PMC 2013 September 01. 